\title{
Vibrational Spectra and Force Fields of Benzylthiocyanate, Benzylselenocyanate and Benzyltellurocyanate
}

\author{
CARL EINAR SJøGREN
}

Department of Chemistry, University of Oslo, P.O. Box 1033, Blindern, N-0315 Oslo 3, Norway

Infrared and Raman spectra of benzylthiocyanate, benzylselenocyanate and benzyltellurocyanate in the solid phase were recorded and tentative assignments, based upon force fields transferred from similar molecules, were proposed.

A number of organic thio- and selenocyanates have been studied by means of vibrational spectroscopy, ${ }^{1-6}$ but so far very little spectroscopic work exists on organic tellurocyanates. Microwave spectroscopic studies of the ethylthio- ${ }^{7}$ and ethylselenocyanate ${ }^{8}$ indicate that the preferred conformation of the linear XCN group is syn-clinal (gauche) to the methyl group. An $\mathrm{X}$-ray study ${ }^{9}$ of 4-nitrobenzylthiocyanate, 4-nitrobenzylselenocyanate, 4-nitrobenzyltellurocyanate and benzylselenocyanate (BSeCN) shows that the syn-clinal conformer is also preferred in the crystalline state, and the same trend is expected for benzylthiocyanate (BSCN) and benzyltellurocyanate $(\mathrm{BTeCN})$. The first indication, to our knowledge, that the anti conformer also exists, is a recent $\mathrm{X}$-ray study of phenacylthiocyanate and phenacylselenocyanate, ${ }^{10} \mathrm{PhCOCH}_{2} \mathrm{XCN}$.

Force fields have been developed for the methyl ${ }^{11}$ and ethylthiocyanates ${ }^{12}$ and the latter has served as a starting point for the calculations presented here. The aim of this work has been to ascertain whether reliable force fields for the title compounds could be compiled from the force fields of similar, but simpler, molecules. If so, these transferred force fields could be used as an aid in the interpretation of the complex vibrational spectra of BSCN, BSeCN and BTeCN.

\section{EXPERIMENTAL}

The preparation and purification of the title compounds together with some of their physical data, have been described previously. ${ }^{13,14}$

Infrared spectra of BSCN, BSeCN and $\mathrm{BTeCN}$ in the solid phase were recorded on a PerkinElmer model 225 grating spectrometer $\left(4000-400 \mathrm{~cm}^{-1}, \mathrm{KBr}\right.$ pellet) and a Bruker IFS $114 \mathrm{C}$ evacuable Fourier Transform spectrometer (700-40 cm $\mathrm{cm}^{-1}$, polyethylene pellet). Due to their low solubility solution spectra of BSCN, BSeCN and $\mathrm{BTeCN}$ were not obtained. The Fourier Transform spectrometer was equipped with a standard DTGS detector and Mylar film beamsplitters of 3.5 and $12 \mu \mathrm{m}$ thickness were used.

Raman spectra of the pure BSCN and BSeCN solids were recorded with a Dilor RT 30 spectrometer using the $488.0 \mathrm{~nm}$ line of an argon ion laser for excitation (CRL model $52 \mathrm{G}$ ). Due to its low stability with respect to the formation of elemental tellurium, the Raman spectrum of BTeCN could not be recorded.

\section{RESULTS AND DISCUSSION}

Because of their large size, the title compounds have very complex vibrational spectra with a large number of bands. Table 1 shows the observed wavenumbers together with calculated fundamental frequencies. Parts of the infrared spectra are shown in Figs. 1 to 3 and corresponding Raman spectra are given in Figs. 4 and 5. Bands, not assigned as fundamentals, can readily be ascribed to combination frequencies, as indicated in Table 1.

The majority of the bands coincide in the spectra of the three compounds and these have 


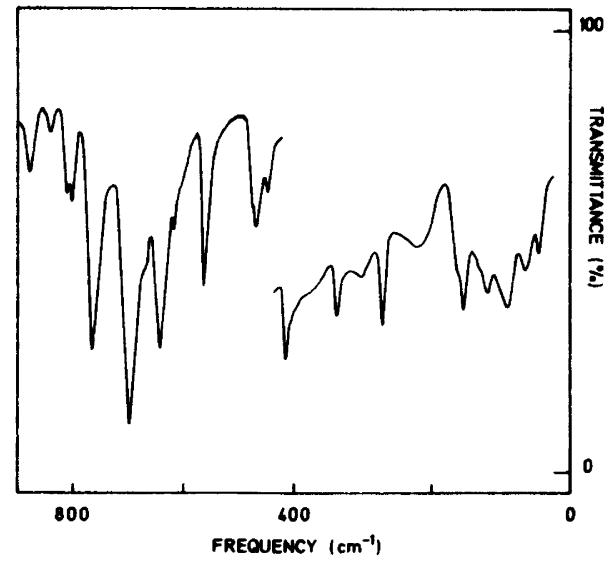

Fig. 1. Infrared spectrum of benzylthiocyanate. $900-400 \mathrm{~cm}^{-1}, \mathrm{KBr}$ pellet; $400-50 \mathrm{~cm}^{-1}$, polyethylene pellet.

been assigned to modes belonging to the common benzyl group. Weak bands in the region $2920-2950 \mathrm{~cm}^{-1}$ were interpreted as the $-\mathrm{CH}_{2}$ stretching modes, and the corresponding $-\mathrm{CH}_{2}$ bendings were assigned to bands at 1422-1425 $\mathrm{cm}^{-1}, 1208-1241 \mathrm{~cm}^{-1}, 1191-1202 \mathrm{~cm}^{-1}$ and $850-886 \mathrm{~cm}^{-1}$ for scissor, twist, wag and rocking modes respectively. A very strong absorption at $2145 \pm 1 \mathrm{~cm}^{-1}$, present in all spectra of BSCN, $\mathrm{BSeCN}$ and $\mathrm{BTeCN}$, was attributed to the $\mathrm{CN}$ stretching mode.

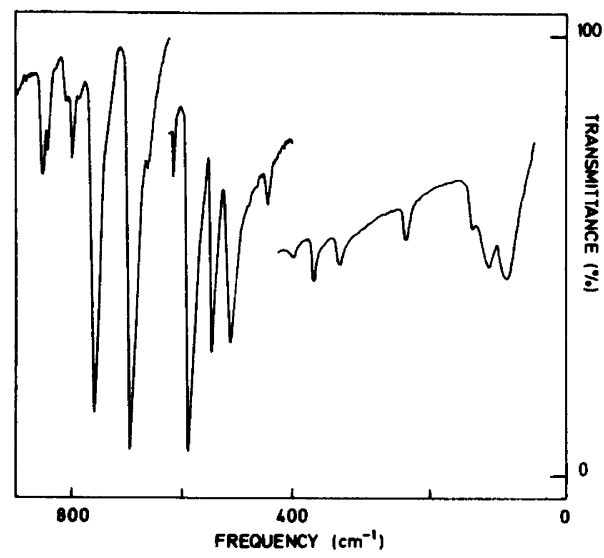

Fig. 2. Infrared spectrum of benzylselenocyanate. $900-400 \mathrm{~cm}^{-1}, \mathrm{KBr}$ pellet; $400-50 \mathrm{~cm}^{-1}$, polyethylene pellet.

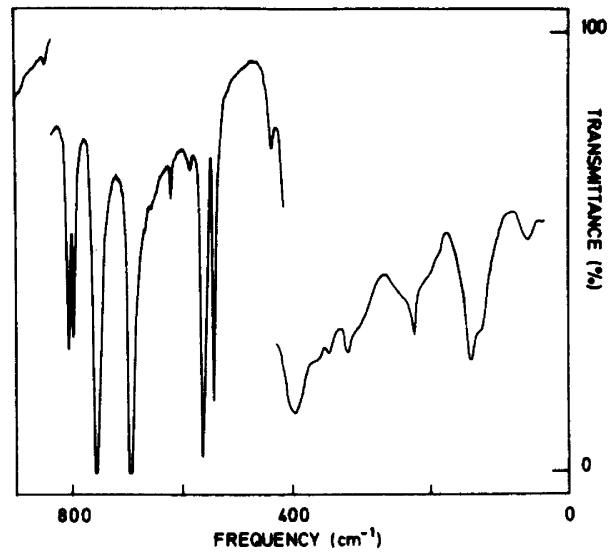

Fig. 3. Infrared spectrum of benzyltellurocyanate. $900-400 \mathrm{~cm}^{-1}, \mathrm{KBr}$ pellet; $400-50 \mathrm{~cm}^{-1}$, polyethylene pellet.

The region above $800 \mathrm{~cm}^{-1}$ could thus be assigned fairly easily by comparing the spectra of the three compounds and by comparing the present normal coordinate calculations, and in particular potential energy distributions and $L_{-}$matrices, with corresponding calculations on benzene ${ }^{15}$ and $p$-fluorobenzonitrile. ${ }^{16}$

The interpretation of the spectral region below $800 \mathrm{~cm}^{-1}$ proved more difficult. Two stretching fundamentals are expected in this region, the XC stretching mode of the $\mathrm{XCN}$ group and the stretching of the $\mathrm{C}_{\alpha} \mathrm{X}$ bond linking the benzyl and $\mathrm{XCN}$ fragments. Force constant calculations immediately showed that whereas the XC vibration can be regarded as a group frequency, showing little or no mixing with other vibrations, the $\mathrm{C}_{\alpha} \mathrm{X}$ stretching, on the other hand, contributes significantly to several fundamentals of the molecules studied here.

A previous study of a series of alkylselenocyanates ${ }^{6}$ including phenylselenocyanate, ${ }^{5,17}$ with $\mathrm{SeC}$ stretching frequencies ranging from $512 \mathrm{~cm}^{-1}$ to $522 \mathrm{~cm}^{-1}$, confirms the interpretation of a $\mathrm{BSeCN}$ band at $516 \mathrm{~cm}^{-1}$ as the SeC stretching fundamental. Likewise, spectra of the tellurocyanate ion $^{18}$ confirm the assignment of a BTeCN band at $438 \mathrm{~cm}^{-1}$ to the corresponding TeC stretching mode. Some ambiguities still remain in the case of the thiocyanates, whether the SC stretching fundamental is to be assigned to the higher of the lower of two bands observed in the $600-700 \mathrm{~cm}^{-1}$ range. ${ }^{6,14}$ This question does 


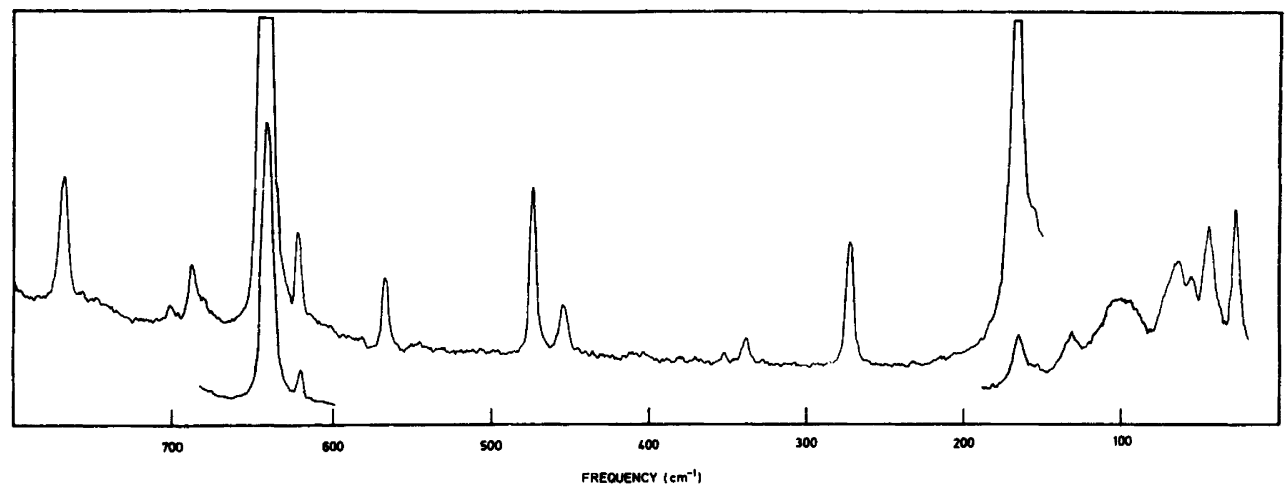

Fig. 4. Raman spectrum of solid benzylthiocyanate.

not arise in the case of $\mathrm{BSCN}$ since, for reasons outlined above, the very intense band at $642 \mathrm{~cm}^{-1}$ can only be interpreted as the SC stretching mode. As shown by the calculation, the $\mathrm{C}_{\alpha} \mathrm{S}$ bond stretching vibration mixes into fundamentals both at lower and higher wavenumbers than the $\mathrm{SC}$ mode.

The XCN linear bending vibrations have been assigned at higher wavenumbers than the $\mathrm{CCC}_{\alpha}$ and $\mathrm{CC}_{\alpha} \mathrm{X}$ bendings, in accordance with previous normal coordinate calculations on methyl ${ }^{11}$ and ethylthiocyanate. ${ }^{12}$

The bending modes below $350 \mathrm{~cm}^{-1}$ have all been calculated at consistently lower wavenumbers than the observations, indicating that the corresponding force constants should be increased. Due to the appearance of interfering lattice modes, the $\mathrm{CC}_{\alpha}$ and $\mathrm{C}_{\alpha} \mathrm{X}$ torsional modes have not been assigned.

\section{FORCE CONSTANT CALCULATIONS}

A force field for $p$-fluorobenzonitrile ${ }^{16}$ based upon an $a b$ initio calculated force field for benzene ${ }^{15}$ was chosen as a basis for the present normal coordinate analysis. Standard bond lengths ${ }^{19}\left(r_{\mathrm{CC}}=139.7 \mathrm{pm}\right.$ and $\left.r_{\mathrm{CH}}=108.4 \mathrm{pm}\right)$ were chosen for the phenyl group, thus allowing direct comparison with previously calculated benzene fundamentals. A tetrahedral configuration around the benzyl $\left(\mathrm{C}_{\alpha}\right)$ carbon was assumed with a $\mathrm{CH}$ bond length of $109.4 \mathrm{pm}$. The remaining geometrical parameters were taken from X-ray data. ${ }^{9}$ In the case of BSCN and $\mathrm{BTeCN}$, structure data were transferred from the corresponding 4-nitro compound. ${ }^{9}$

Force fields for $p$-fluorobenzonitrile, ${ }^{16}$ including the $\mathrm{CC}$ (ring) $\mathrm{C}_{\alpha}$ bend force constant, toluene $^{20}$ and ethylthiocyanate ${ }^{12}$ were transferred to

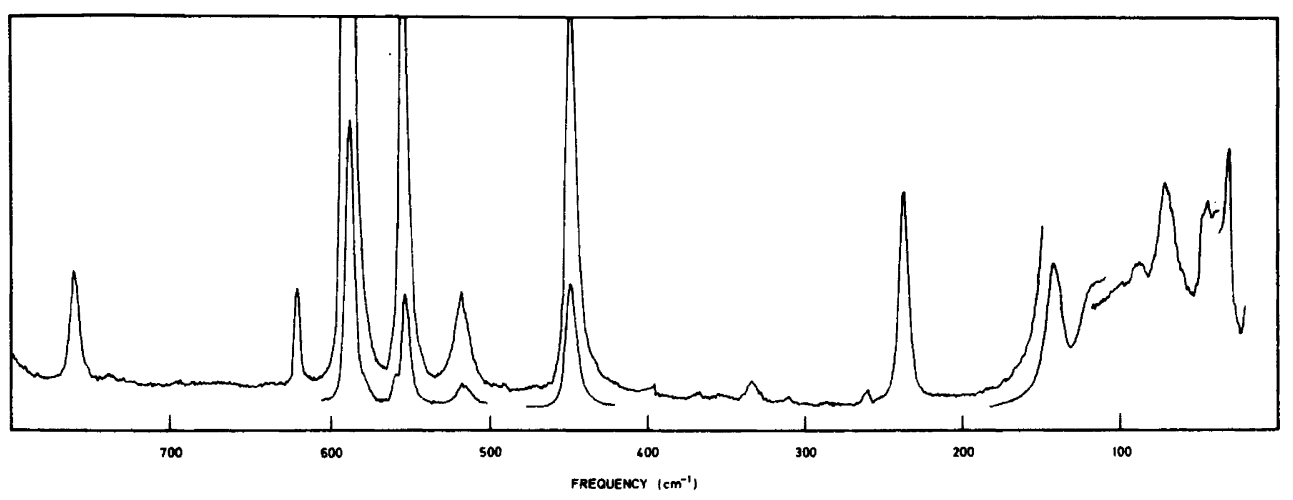

Fig. 5. Raman spectrum of solid benzylselenocyanate.

Acta Chem. Scand. A 38 (1984) No. 9 
Table 1. Infrared and Raman spectral data ${ }^{a}$ and calculated fundamental frequencies $\left(\mathrm{cm}^{-1}\right)$ of BSCN, $\mathrm{BSeCN}$ and $\mathrm{BTeCN}$.

\begin{tabular}{|c|c|c|c|c|c|c|c|c|c|}
\hline \multicolumn{3}{|c|}{ BSCN } & \multicolumn{3}{|c|}{$\mathrm{BSeCN}$} & \multicolumn{3}{|c|}{ BTeCN } & \multirow[b]{2}{*}{ Assignment } \\
\hline Obs. & $\mathbf{R} / \mathbf{I R}^{b}$ & Calc. & Obs. & $\mathbf{R} / \mathbf{I R}$ & Calc. & Obs. & IR & Calc. & \\
\hline 3106 & $-/ \mathbf{v w}^{c}$ & 3092 & 3094 & $-/ \mathrm{vw}$ & 3092 & 3096 & vw & 3092 & i.p. ${ }^{d}$ \\
\hline 3087 & $-/ w$ & 3081 & 3080 & $-/ \mathrm{vw}$ & 3081 & 3077 & $\mathbf{w}$ & 3081 & i.p. \\
\hline 3064 & $w / w$ & 3072 & 3066 & w/vw & 3072 & & & 3072 & i.p. \\
\hline 3058 & vs/- & 3064 & 3059 & $\mathrm{~m} / \mathrm{vw}$ & 3064 & 3056 & $\mathbf{w}$ & 3064 & i.p. \\
\hline 3045 & $\mathbf{w} /-$ & 3055 & 3040 & $\mathbf{w} /-$ & 3055 & & & 3055 & i.p. \\
\hline 3032 & $-/ \mathrm{m}$ & & 3026 & $-/ w$ & & 3025 & $\mathbf{m}$ & & comb. \\
\hline 3008 & $-/ v w$ & & 3000 & $w / w$ & & 2998 & $\mathbf{w}$ & & comb. \\
\hline 2991 & $\mathrm{~m} / \mathrm{vw}$ & & & & & 2993 & w & & comb. \\
\hline & & & 2978 & vw/vw sh & & 2962 & vw & & comb. \\
\hline 2948 & $s / w$ & 2949 & 2952 & $\mathrm{~s} / \mathrm{vw}$ & 2948 & 2944 & vw & 2948 & $-\mathrm{CH}_{2}$ asym. stretch \\
\hline 2925 & $-/ \mathrm{vw}$ sh & 2919 & 2924 & $-/ v w$ & 2919 & 2922 & vw & 2919 & $-\mathrm{CH}_{2}$ sym. stretch \\
\hline 2145 & vs/vs & 2170 & 2146 & vs/vs & 2161 & 2146 & vs & 2143 & CN stretch \\
\hline 1602 & $\mathbf{s} / \mathbf{w}$ & 1625 & 1598 & vs/w & 1625 & 1597 & $\mathbf{s}$ & 1625 & i.p. \\
\hline 1585 & $\mathbf{w} / \mathbf{w}$ & 1618 & 1582 & $\mathbf{w} / \mathbf{w}$ & 1618 & 1581 & vw & 1618 & i.p. \\
\hline 1548 & $-/ \mathbf{v w}$ & & 1540 & $-/ v w$ & & $1558-1$ & $495^{e}$ & & comb. \\
\hline 1491 & $-/ \mathbf{s}$ & 1500 & 1492 & $w / s$ & 1500 & 1491 & $\mathbf{s}$ & 1500 & i.p. \\
\hline 1452 & $-/ \mathbf{s}$ & 1437 & 1454 & w/vs & 1437 & 1453 & $\mathbf{s}$ & 1437 & i.p. \\
\hline 1425 & $\mathrm{~m} / \mathrm{s}$ & 1426 & 1424 & $w / s$ & 1424 & 1422 & $\mathbf{w}$ & 1427 & $\mathrm{CH}_{2}$ scissor \\
\hline 1393 & $-/$ vw & & 1389 & $-/ w$ & & 1387 & vw & & comb. \\
\hline & & & 1362 & $-/ w$ & & & & & comb. \\
\hline 1336 & $-/ v w$ & 1329 & 1335 & $-/ w$ & 1330 & 1333 & $\mathbf{w}$ & 1327 & i.p. \\
\hline $\begin{array}{l}1323 \\
1288\end{array}$ & $\begin{array}{l}-/ \mathbf{w} w \\
\mathbf{v w} / \mathbf{w}\end{array}$ & & & & & 1318 & vw & & comb. \\
\hline $\begin{array}{l}1288 \\
1255\end{array}$ & $\begin{array}{l}\mathbf{v w} / \mathbf{w} \\
\mathrm{sh} /-\end{array}$ & $\begin{array}{l}1296 \\
1255\end{array}$ & $\begin{array}{l}1281 \\
1252\end{array}$ & $\begin{array}{l}\mathbf{v w} / \mathbf{v w} \\
\mathbf{w} /-\end{array}$ & $\begin{array}{l}1296 \\
1250\end{array}$ & 1301 & vw & $\begin{array}{l}1293 \\
1250\end{array}$ & $\begin{array}{l}\text { 1.p. } \\
\text { C. }\end{array}$ \\
\hline 1241 & $\mathbf{s} / \mathbf{s}$ & 1224 & 1217 & $\mathbf{s} / \mathbf{s}$ & 1222 & 1208 & $\mathbf{w}$ & 1218 & $\mathrm{CH}_{2}$ twist \\
\hline 1202 & $\mathrm{~s} / \mathrm{m}$ & 1200 & 1191 & w/vs & 1202 & & & 1199 & $\mathrm{CH}_{2}$ wag \\
\hline 1183 & $\mathrm{~m} / \mathrm{vw}$ & 1174 & 1176 & $\mathbf{m} / \mathbf{m}$ & 1174 & 1176 & vw & 1174 & i.p. \\
\hline 1173 & $\mathbf{w} /-$ & & 1169 & $-/$ vw sh & & & & & comb. \\
\hline $\begin{array}{l}1158 \\
1147\end{array}$ & $\begin{array}{l}m / w \\
-/ w\end{array}$ & 1162 & 1156 & $\mathrm{~m} / \mathrm{m}$ & 1162 & 1155 & vw & 1162 & i.p. \\
\hline & $T \mathrm{w}$ & & 1131 & $w / w$ & & 1139 & $\mathbf{s}$ & & comb. \\
\hline & & & & & & 1114 & $\mathbf{v w}$ & & comb. \\
\hline 1109 & $-/ w$ & & 1104 & $-/ w$ & & 1103 & vw & & comb. \\
\hline & & & & & & 1084 & vw & & comb. \\
\hline 1072 & $-/ \mathbf{m}$ & 1088 & 1069 & $\mathrm{vw} / \mathrm{s}$ & 1088 & 1059 & $\mathbf{s}$ & 1088 & i.p. \\
\hline 1025 & $\mathbf{m} / \mathbf{w}$ & 1022 & 1027 & $\mathbf{m} / \mathbf{m}$ & 1022 & $\begin{array}{l}1028 \\
1020\end{array}$ & $\begin{array}{l}\text { m } \\
\text { vw sh }\end{array}$ & 1022 & $\begin{array}{l}\text { i.p. } \\
\text { comb. }\end{array}$ \\
\hline 1003 & vs/vw & 990 & 1002 & $\mathrm{vs} / \mathrm{m}$ & 990 & 1001 & vw & 990 & i.p. \\
\hline 990 & $\mathrm{vw} / \mathrm{vw}$ & 982 & 990 & $\mathbf{v w} /-$ & 982 & 996 & vw & 982 & o.o.p. \\
\hline 965 & $-/ v w$ & 964 & 966 & $-/ w$ & 964 & 963 & $\mathbf{w}$ & 964 & $\begin{array}{l}\text { comb. } \\
\text { o.o.p. }\end{array}$ \\
\hline 918 & $-/ \mathrm{m}$ & 906 & 916 & $\mathrm{vw} / \mathrm{m}$ & 905 & 912 & m & 904 & o.o.p. \\
\hline 886 & $\mathrm{vw} / \mathrm{m}$ & 840 & 855 & $\mathrm{vw} / \mathrm{m}$ & 819 & 850 & vw & 829 & $\mathrm{CH}_{2}$ rock \\
\hline 850 & $v w / v w$ & 839 & 846 & $v w / w$ & 839 & & & 839 & $0.0 . p$. \\
\hline 814 & $\mathbf{w} / \mathbf{m}$ & 814 & 814 & $\mathbf{w} / \mathbf{v w}\}$ & 809 & 806 & $\mathbf{m}\}$ & 808 & i $p$ \\
\hline 803 & $\mathbf{m} / \mathbf{m} /$ & 014 & 802 & $\mathbf{w} / \mathbf{w}$ & 007 & 798 & $\mathrm{~m} /$ & 000 & \\
\hline 767 & $\mathrm{~m} / \mathrm{s}$ & $783(37)^{f}$ & $f 759$ & $\mathrm{~m} / \mathrm{vs}$ & $744(3)$ & 756 & vs & $741(1)$ & o.o.p./ $\mathrm{C}_{\alpha} \mathrm{X}$ stretch \\
\hline 695 & w/vs & $688(5)$ & $\begin{array}{l}694 \\
664\end{array}$ & $\begin{array}{l}\mathbf{v w / v s} \\
-/ \mathbf{w}\end{array}$ & 690 & $\begin{array}{l}694 \\
654\end{array}$ & $\begin{array}{l}\text { vs } \\
\text { vw sh }\end{array}$ & 690 & $\begin{array}{l}\text { o.o.p. } \\
\text { comb. }\end{array}$ \\
\hline 642 & vs/vs & 641 & 516 & $\mathrm{~m} / \mathrm{m}$ & $518(7)$ & 438 & w & 433 & XC stretch \\
\hline
\end{tabular}


Table 1. Continued.

\begin{tabular}{|c|c|c|c|c|c|c|c|c|}
\hline $\mathrm{m} / \mathrm{vw}$ & 619 & 620 & $\mathrm{~m} / \mathrm{w}$ & 619 & 620 & $\mathbf{w}$ & 619 & i.p. \\
\hline $\mathrm{m} / \mathrm{vw} \mathrm{sh}$ & $702(22)$ & 592 & vs/vs & $614(28)$ & 564 & s & 591 (19) & o.o.p./C $\mathrm{C} \alpha$ stretch \\
\hline 565 & $554(6)$ & 550 & $\mathrm{~s} / \mathrm{m}$ & $540(7)$ & 542 & m & $520(14)$ & i.p. $/ \mathrm{C}_{\alpha} \mathrm{X}$ stretch \\
\hline$-/ \mathrm{vw}$ & & 481 & $-/ v w$ & & & & & comb. \\
\hline $\begin{array}{l}\mathrm{l} / \mathrm{s} \\
\mathrm{m} / \mathrm{m}\end{array}$ & $\begin{array}{l}444 \\
427(6)\end{array}$ & $\begin{array}{l}448 \\
436\end{array}$ & $\begin{array}{l}\mathrm{s} / \mathrm{w} \\
\mathrm{vw} \mathrm{sh} /-\end{array}$ & $411(17)$ & 397 & vs & $400(20)$ & o.o.p./ $\mathrm{C}_{\alpha} \mathrm{X}$ stretch \\
\hline$-/ \mathrm{s}$ & 401 & 398 & $-/ w$ & 401 & & & 401 & o.o.p. \\
\hline $\begin{array}{l}-/ \mathbf{w} \\
\mathrm{vw} /-\end{array}$ & 399 & 368 & $-/ \mathrm{m}$ & 378 & $\begin{array}{l}366 \\
348\end{array}$ & $\begin{array}{l}\mathrm{m} \text { sh } \\
\mathrm{m}\end{array}$ & 367 & $\begin{array}{l}\mathrm{XCN} \text { linear bend } \\
\text { comb. }\end{array}$ \\
\hline $\mathrm{vw} / \mathrm{m}$ & 285 & 332 & $\mathbf{w} / \mathbf{w}$ & 285 & 320 & & 287 & $\mathrm{CCC}_{\alpha}$ bend \\
\hline $\begin{array}{l}-/ \mathrm{vw} \\
\mathrm{m} / \mathrm{s}\end{array}$ & $256(10)$ & 311 & $\mathbf{v w} /-$ & 301 & 303 & vw sh & $186(51)$ & comb. \\
\hline$-/ w b$ & $200(17)$ & 261 & $\mathbf{w} /-$ & & & & & $\begin{array}{l}\text { comb. } \\
\text { cols }\end{array}$ \\
\hline $\begin{array}{l}\mathrm{s} / \mathrm{w} \text { sh } \\
\mathrm{sh} / \mathrm{s}\end{array}$ & 143 & 138 & $\mathrm{~m} / \mathrm{s}$ & 127 & $\begin{array}{l}128 \\
143\end{array}$ & $\begin{array}{l}\mathbf{s} \\
\mathbf{s}\end{array}$ & 115 & $\begin{array}{l}\mathrm{C}_{\alpha} \mathrm{XC} \text { bend } \\
\text { lattice mode }\end{array}$ \\
\hline $\begin{array}{l}\mathrm{s} / \mathrm{m} \\
\mathrm{b} / \mathrm{s} \\
\mathrm{s} / \mathrm{m} \\
\mathrm{m} /- \\
\mathrm{s} / \mathrm{m} \\
\mathrm{vs} /-\end{array}$ & 101 & $\begin{array}{r}116 \\
89 \\
73 \\
46 \\
38 \\
32\end{array}$ & $\begin{array}{l}-/ \mathrm{m} \\
\mathrm{w} \mathrm{b} / \mathrm{m} \\
\mathrm{m} /- \\
\mathrm{m} /- \\
\mathrm{m} /- \\
\mathrm{m} /-\end{array}$ & 84 & 57 & $\mathrm{~m}$ & 76 & $\begin{array}{l}\text { lattice mode } \\
\mathrm{CC}_{\alpha} \text { out of plane } \\
\text { lattice mode } \\
\text { lattice mode } \\
\text { lattice mode } \\
\text { lattice mode }\end{array}$ \\
\hline & $\begin{array}{r}38 \\
4\end{array}$ & & & $\begin{array}{r}35 \\
4\end{array}$ & & & $\begin{array}{r}31 \\
4\end{array}$ & $\begin{array}{l}\mathrm{C}_{\alpha} \mathrm{X} \text { torsion } \\
\mathrm{CC}_{\alpha} \text { torsion }\end{array}$ \\
\hline
\end{tabular}

${ }^{a}$ Bands in the regions $4000-3200,2900-2200$ and $2100-1650 \mathrm{~cm}^{-1}$ were omitted. ${ }^{b}$ Tabulated values are infrared frequencies, except for bands of very low intensity in infrared spectra. ${ }^{c}$ Abbreviations: $s$, strong; $\mathrm{m}$, medium; w, weak; v, very; b, broad; sh, shoulder. ${ }^{d}$ i.p.: Benzene in plane mode, o.o.p.: Benzene out of plane mode. ${ }^{e}$ Several very weak bands observed in this interval. $f$ Numbers in italics denote fundamentals containing $\mathrm{C}_{\alpha} \mathrm{X}$ stretching, values in parentheses are approximate $\mathrm{C}_{\alpha} \mathrm{X}$ potential energy contribution.

the phenyl, $\mathrm{CH}_{2}$ and $\mathrm{SCN}$ groups, giving the calculated frequencies shown in Table 1 . Since normal coordinate calculations on ethylselenocyanate and ethyltellurocyanate have not been available, reasonable approximations had to be found for the $\mathrm{XCN}$ force fields of $\mathrm{BSeCN}$ and BTeCN. As a first approximation the ethylthiocyanate force constants were transferred to $\mathrm{BSeCN}$ and $\mathrm{BTeCN}$ after being scaled according to the relation: $f_{\mathrm{xy}} \cdot r_{\mathrm{x}} \cdot r_{\mathrm{y}}=$ constant, where $f_{\mathrm{xy}}$ is the force constant in $\mathrm{N} / \mathrm{m}$ and $r_{\mathrm{x}}$ and $r_{\mathrm{y}}$ are bond lengths. This simple relationship has previously been successfully applied to a series of halogenated cyclohexanes. ${ }^{21}$ It was found that, although the scaling works fairly well for the localized $\mathrm{XCN}$ modes, the $\mathrm{C}_{\alpha} \mathrm{X}$ and $\mathrm{XC}$ stretching force constants had to be treated differently.

In the case of $\mathrm{BSeCN}$ the $\mathrm{SeC}$ and $\mathrm{C}_{\alpha} \mathrm{Se}$ the force constants were refined in a normal coordinate calculation on ethylselenocyanate, using frequencies from the literature. ${ }^{6}$ The rest of the force field, including the $\mathrm{SeC} / \mathrm{C}_{\alpha} \mathrm{Se}$ stretch-stretch interaction, was transferred, after appropriate scaling, from ethyl thiocyanate both in the case of ethylselenocyanate and $\mathrm{BSeCN}$. The final values were somewhat different from the scaled thiocyanate force constants (refined: $f_{\mathrm{C}_{\mathrm{B}} \mathrm{se}}=156.9 \mathrm{~N} / \mathrm{m}, f_{\mathrm{SeC}}=340.4 \mathrm{~N} / \mathrm{m}$; scaled: $\left.f_{\mathrm{C}_{u} \mathrm{Se}}=226 \mathrm{~N} / \mathrm{m}, f_{\mathrm{SeC}}=314 \mathrm{~N} / \mathrm{m}\right)$.

In the case of $\mathrm{BTeCN}$ a similar approach was employed, the main difference being that the $\mathrm{TeC}$ stretching force constant could be transferred from ethylthiocyanate along with the rest of the SCN force field. The scaled $f_{\mathrm{TeC}}=247 \mathrm{~N} / \mathrm{m}$ can be compared with the corresponding value from the tellurocyanate ion, ${ }^{18} 269 \mathrm{~N} / \mathrm{m}$.

The final BTeCN force field includes a $\mathrm{C}_{\alpha} \mathrm{Te}$ stretching force constant transferred from $\mathrm{BSeCN}$ and scaled according to the relation above. The force fields for the $\mathrm{XCN}$ groups, in their final form, are given in Table 2.

As apparent from Table 1 the correspondence 
Table 2. Force constants of the $-\mathrm{CH}_{2} \mathrm{XCN}$ group. ${ }^{a}$

$\begin{array}{llll}\begin{array}{l}\text { Force } \\ \text { constant }\end{array} \text { BSCN } \quad \text { BSeCN } \quad \text { BTeCN } & \text {. }\end{array}$

Stretch and stretch-stretch; $\mathrm{Nm}^{-1}$

$\begin{array}{lccc}f_{\mathrm{CN}} & 1673 & 1673^{b} & 1673^{b} \\ f_{\mathrm{XC}} & 372 & 340.4^{c} & 247 \\ f_{\mathrm{C}_{b} \mathrm{X}} & 265 & 156.9^{\mathrm{c}} & 130^{\mathrm{d}} \\ f_{\mathrm{C}_{u} \mathrm{H}} & 469.6^{e} & 469.6^{e} & 469.6^{e} \\ f_{\mathrm{CC}_{\mathrm{u}}} & 468.13^{e} & 468.13^{e} & 468.13^{e} \\ f_{\mathrm{C}_{u} \mathrm{H} / \mathrm{C}_{u} \mathrm{H}} & 7.3^{e} & 7.3^{e} & 7.3^{e} \\ f_{\mathrm{C}_{u} \mathrm{X} / \mathrm{XC}} & 30 & 25 & 21\end{array}$

Bend and bend-bend; $\mathrm{aN} \cdot \mathrm{m} \cdot \mathrm{rad}^{-2}$

$\begin{array}{llll}f_{\mathrm{CC}_{a} \mathrm{X}} & 1.16 & 1.16 & 1.16 \\ f_{\mathrm{C}_{\mathrm{a}} \mathrm{XC}} & 0.68 & 0.68 & 0.68 \\ f_{\mathrm{HC}} \mathrm{X} & 0.66 & 0.66 & 0.66 \\ f_{\mathrm{XCN}} & 0.37^{f} & 0.37^{f} & 0.37^{f} \\ f_{\mathrm{CC}_{u} \mathrm{H}} & 0.6417^{e} & 0.6417^{e} & 0.6417^{e} \\ f_{\mathrm{HC}} \mathrm{H} & 0.5351^{e} & 0.5351^{e} & 0.5351^{e} \\ f_{\mathrm{HC}_{a} \mathrm{X} / \mathrm{HC}_{\mathrm{a}} \mathrm{X}} & -0.125 & -0.125 & -0.125 \\ f_{\mathrm{HC}_{a} \mathrm{X} / \mathrm{CC}_{a} \mathrm{H}} & -0.015 & -0.015 & -0.015 \\ f_{\mathrm{CC}_{a} \mathrm{H} / \mathrm{CC}_{u} \mathrm{H}} & -0.0211^{e} & -0.0211^{e} & -0.0211^{e}\end{array}$

Stretch-bend; $\mathrm{nN} \cdot \mathrm{rad}^{-1}$

$\begin{array}{llll}f_{\mathrm{C}_{u} \mathrm{X} / \mathrm{HC}_{\mathrm{u}} \mathrm{x}} & 3.5 & 3.2 & 2.9 \\ f_{\mathrm{CC}_{a} / \mathrm{CC}_{a} \mathrm{H}} & 2.335^{e} & 2.335^{e} & 2.335^{e}\end{array}$

Torsion; aN $\cdot \mathrm{m} \cdot \mathrm{rad}^{-2}$

$\begin{array}{llll}f_{\mathrm{C}_{a} \mathrm{X}-\tau} & 0.02 & 0.02 & 0.02 \\ f_{\mathrm{CC}_{u}-\tau} & 0.0001^{e} & 0.0001^{e} & 0.0001^{e}\end{array}$

${ }^{a}$ Transferred from $\mathrm{CH}_{3} \mathrm{CH}_{2} \mathrm{SCN}$ (Ref. 12) and scaled according to $f_{\mathrm{xy}} \cdot r_{\mathrm{x}} \cdot r_{\mathrm{y}}=$ constant, except where noted. ${ }^{b}$ Transferred from $\mathrm{CH}_{3} \mathrm{CH}_{2} \mathrm{SCN}$ (Ref. 12) without scaling. ${ }^{c}$ Transferred from $\mathrm{CH}_{3} \mathrm{CH}_{2} \mathrm{SeCN}$, see text. ${ }^{d}$ Transferred from $\mathrm{BSeCN}$ with scaling. ${ }^{e}$ Transferred from toluene (Ref. 20). ${ }^{f}$ Mean of two values reported for $\mathrm{CH}_{3} \mathrm{CH}_{2} \mathrm{SCN}$ (Ref. 12).

between observed and calculated low frequency fundamentals leaves room for improvement. In particular, the modes involving $\mathrm{C}_{\alpha} \mathrm{X}$ stretch show some deviations which could possibly be removed by refining the interaction force constants coupling the $\mathrm{XCN}$ and benzyl vibrations.

Where little or no mixing occurs between vibrations of the benzyl and XCN fragments, the transferred force field yields an acceptable correspondence between observed and calculated fundamentals.
Acknowledgements. Thanks are due to J. Songstad, Bergen, for supplying the samples and to C. J. Nielsen for helpful discussions.

\section{REFERENCES}

1. Hirschmann, R. P., Kniseley, R. N. and Fassel, V. A. Spectrochim. Acta 20 (1964) 809.

2. Moritz, A. G. Spectrochim. Acta 22 (1966) 1021.

3. Crowder, G. A. J. Mol. Spectrosc. 23 (1967) 108.

4. Ellestad, O. H. and Torgrimsen, T. J. Mol. Struct. 12 (1972) 79.

5. Aynsley, E. E., Greenwood, N. N. and Sprague, M. J. J. Chem. Soc. (1965) 2395.

6. Franklin, W. J., Werner, R. L. and Ashby, R. A. Spectrochim. Acta Part A 30 (1974) 387.

7. Bjørseth, A. and Marstokk, K. M. J. Mol. Struct. 11 (1972) 15.

8. Sakaizumi, T., Itakura, T., Ohhashi, O. and Yamaguchi, I. Koen Yoshishu-Bunshi Kozo Sogo Toronkai (Jpn.) (1979) 158.

9. Maartmann-Moe, K., Sanderud, K. and Songstad, J. Acta Chem. Scand. A 38 (1984) 187.

10. Songstad, J. Private communication.

11. Crowder, G. A. J. Mol. Struct. 7 (1971) 147.

12. Bjørseth. A. Acta Chem. Scand. A 28 (1974) 113.

13. Maartmann-Moe, K., Sanderud, K. and Songstad, J. Acta Chem. Scand. A 35 (1981) 151.

14. Maartmann-Moe, K., Sanderud, K. and Songstad, J. Acta Chem. Scand. B 36 (1982) 211.

15. Pulay, P., Fogarasi, G. and Boggs, J. E. J. Chem. Phys. 74 (1981) 3999.

16. Bak, B., Christensen, D. H., Kristiansen, N. A., Nicolaisen, F. and Nielsen, O. F. Acta Chem. Scand. A 37 (1983) 601.

17. Giorganni, S., Passerini, A. and Passerini, R. Spectrosc. Lett. 16 (1983) 775.

18. Klæboe, P., Nielsen, C. J. and Songstad, J. Acta Chem. Scand. A 31 (1977) 884.

19. Langseth, A. and Stoicheff, P. B. Can. J. Phys. 34 (1956) 350.

20. La Lau, C. and Snyder, R. G. Spectrochim. Acta Part A 27 (1971) 2073.

21. Woldbæk, T., Nielsen, C. J. and Klæboe, P. J. Mol. Struct. 66 (1980) 31.

Received March 21, 1984.

Acta Chem. Scand. A 38 (1984) No. 9 